\title{
Review Article \\ The Effects of Heteroatom Adsorption on the Electronic Properties of Phosphorene
}

\author{
Mengyao Sun, Zhiyong Wang, Yayun Zhao, Junchao Jin, Jianrong Xiao, and Liu Wang \\ College of Science, Guilin University of Technology, Guilin 541004, China \\ Correspondence should be addressed to Zhiyong Wang; zhiyongwang520@gmail.com
}

Received 18 February 2017; Accepted 23 April 2017; Published 24 May 2017

Academic Editor: Mohamed Bououdina

Copyright (c) 2017 Mengyao Sun et al. This is an open access article distributed under the Creative Commons Attribution License, which permits unrestricted use, distribution, and reproduction in any medium, provided the original work is properly cited.

\begin{abstract}
A new 2D material, phosphorene, has several remarkable advantages; various superiorities make phosphorene a research hotspot. This paper provides comprehensive information about the structure and electronic and magnetic properties of phosphorene adsorbed with atoms, including alkali and alkaline-earth metal atoms, nonmetallic atoms, noble metal atoms, and transitionmetal atoms. Phosphorene adsorbed with alkali and alkaline-earth metal atoms, such as Li and $\mathrm{Na}$ adatoms, becomes an n-type semiconductor, while phosphorene adsorbed with $\mathrm{Be}$ and $\mathrm{Mg}$ atoms becomes a p-type semiconductor. In view of nonmetallic adatoms ( $\mathrm{B}, \mathrm{C}, \mathrm{N}$, and $\mathrm{O}$ ), the $\mathrm{B}$ adatom decorated phosphorene becomes metallic, the band gap of phosphorene adsorbed with $\mathrm{C}$ adatom decreases, and the phosphorene is p-type with $\mathrm{N}$ adatom, while the electronic property of $\mathrm{O}$ adatom adsorption case is affected slightly. Regarding noble metal adatoms adsorption condition, the Ag adatom makes phosphorene a n-type semiconductor, the $\mathrm{Au}$ adatom induces phosphorene to have a magnetism of $1 \mu \mathrm{B}$, and the electronic property of phosphorene is changed by adsorbing with $\mathrm{Pt}$ adatom. Among transition-metal adatoms, such as $\mathrm{Fe}, \mathrm{Ni}, \mathrm{Co}, \mathrm{Cu}$, and $\mathrm{Zn}$ adatoms, the band gap is reduced when $\mathrm{Fe} / \mathrm{Ni}$ adatom adheres to the surface of phosphorene, The Co adsorbed phosphorene turns into a polar-gapless semiconductor and phosphorene is proved to be $\mathrm{n}$-type with $\mathrm{Cu}$ adatom, but it is testified that the $\mathrm{Zn}$ atom is not suitable to adsorb on the phosphorene.
\end{abstract}

\section{Introduction}

In recent years, phosphorene has caused widespread interest following the discovery of graphene [1] and the transitionmetal dichalcogenide [2] (TMDC) family. But it has not attracted any attention when discovered by physicists and chemists in 1914 due to its instable structure and strong toxicity [3]. The quest of searching for two-dimensional (2D) with more significant key properties, such as the high carrier mobility and remarkable band gap, has made phosphorene a new member of 2D material family. Since the high-performance field-effect transistors (FETs) based on phosphorene have been fabricated in 2014 [4], abundant theoretical and experimental researches about the physical, chemical, and electronic properties of phosphorene have been made and led to the manufacturing of phosphorenebased nanodevices in electronics, optoelectronics, and photovoltaics [5].

Phosphorene is mechanically exfoliated from bulk black phosphorus $[6,7]$. The reason why the properties of phosphorene are so novel is that it has a strong covalent bonding system but weak van der Waals (vdWs) interlayer interactions [8]. Figures 1(a)-1(c) show the optimized structures of phosphorene, whose atoms are arranged into a wrinkled honeycomb lattice structure with an up (U) and down (D) sequence of UUUDDD $[9,10]$. From Figures 1(b) and 1(c), it can be found that the orthogonal lattice parameters of phosphorene are $a_{1}=3.35 \AA, a_{2}=4.62 \AA$, and $a_{3}=2.11 \AA$ [11], which is the thickness of phosphorene. Figure $1(\mathrm{~d})$ shows that phosphorene is a p-type semiconductor with a direct band gap of $1.5 \mathrm{eV}$ [12]. Interestingly, it can be noted that the band gap of phosphorene decreased with the increased number of layers, as shown in Figure 1(e). Furthermore, the energy gap $\left(E_{\mathrm{g}}\right)$ also varies as the opposite number of layers, revealing that the change of layers can tune the electronic properties of phosphorene [13]. Figure 1(f) shows that the energy also depends on in-plane strain along the $x$ and $y$ direction [14-16]. In particular, the strain of $5 \%$ and more can change phosphorene from a direct-gap to an indirect-gap semiconductor [9].

As a new 2D material, phosphorene, has attracted more attention than other 2D materials such as graphene [4], $\mathrm{MoS}_{2}$ 


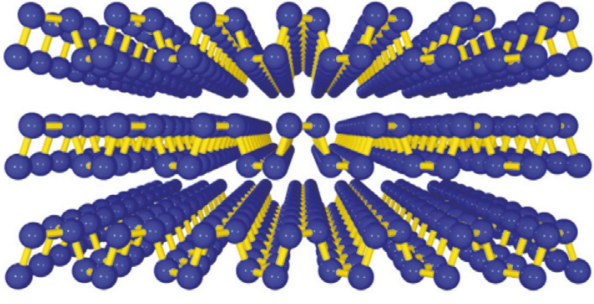

(a)

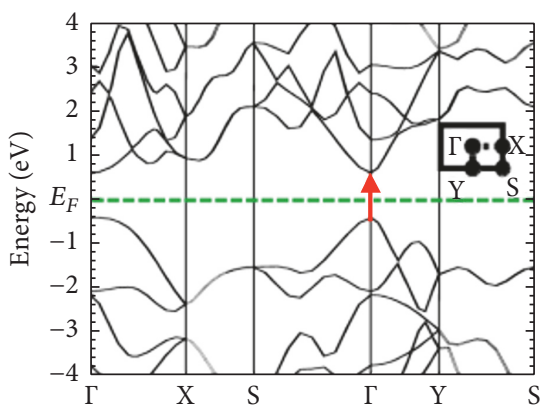

(d)

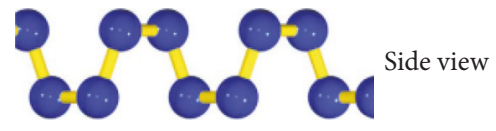

(b)

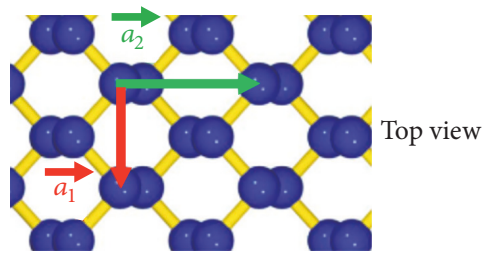

(c)

Number of layers $N$

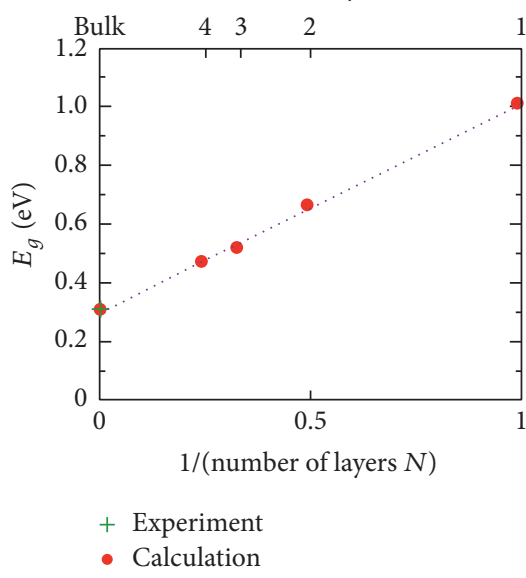

(e)

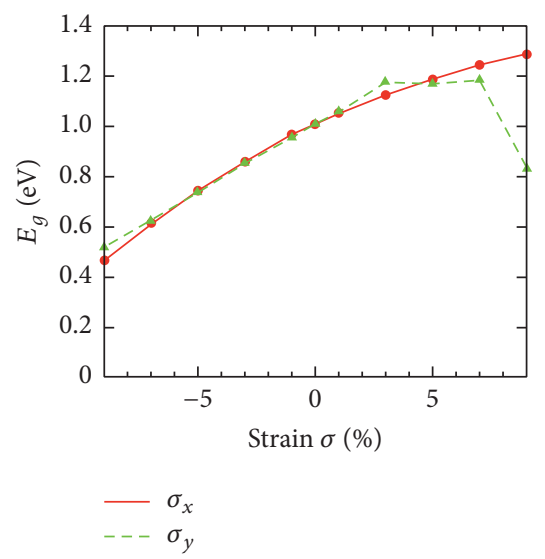

(f)

Figure 1: (a) Perspective side view of phosphorene. (b, c) Side and top views of phosphorene. (d) Band structure of phosphorene monolayer. $(\mathrm{e}, \mathrm{f})$ Results for the dependence of the energy gap in phosphorene on (e) the number of layers and (f) the strain along the $x$ and $y$ direction [9].

TABLE 1: Fundamental properties of different 2D materials [19].

\begin{tabular}{|c|c|c|c|c|c|}
\hline Type & $\begin{array}{l}\text { Phosphorene } \\
\text { Semiconductor }\end{array}$ & $\begin{array}{l}\text { Graphene } \\
\text { Semimetal }\end{array}$ & $\begin{array}{c}\mathrm{MoS}_{2} \\
\text { Semiconductor }\end{array}$ & $\begin{array}{c}\text { WSe }_{2} \\
\text { Semiconductor }\end{array}$ & $\begin{array}{c}h \text {-BN } \\
\text { Insulator }\end{array}$ \\
\hline Bandgap (eV) & $0.3-2.0$ & 0 & $1.2-1.8$ & $1.2-1.7$ & 5.9 \\
\hline $\begin{array}{l}\text { Carrier mobility, } \\
\left(\mathrm{cm}^{2} \mathrm{~V}^{-1} \mathrm{~s}^{-1}\right)\end{array}$ & $\approx 1000$ & $\approx 2 \times 10^{5}$ & $10-200$ & $140-500$ & - \\
\hline On/off ratio & $10^{3}-10^{5}$ & $5.5-44$ & $10^{6}-10^{8}$ & $10^{4}-10^{6}$ & - \\
\hline $\begin{array}{l}\text { Thermal conductance } \\
\left(\mathrm{Wm}^{-1} \mathrm{~K}^{-1}\right)\end{array}$ & $10-36$ & $\approx 5 \times 10^{3}$ & $34.5-52$ & 9.7 & $250-360$ \\
\hline $\begin{array}{l}\text { Thermoelectric } \\
\text { performance, } \mathrm{ZT}\end{array}$ & $1-2.5$ & $\approx 0$ & 0.4 & 0.91 & - \\
\hline Young's modulus (GPa) & $35-166$ & 1000 & $270 \pm 100$ & 75-195 & $220-880$ \\
\hline Fracture strain (\%) & $24-32$ & $27-38$ & $25-33$ & $26-37$ & 24 \\
\hline Conduction type & Ambipolar & Ambipolar & n-type & Ambipolar & - \\
\hline
\end{tabular}

[45-47], WSe ${ }_{2}$ [48], and h-BN [49-51]. The fundamental properties of different 2D materials including phosphorene have been summed up in Table 1 [19], including outstanding band gap, high carrier mobility and on/off ratio, novel physical phenomena (e.g., superconductivity and quantum Hall effect [52]), distinct mechanical characters (anisotropic Young's modulus [53]), adjustable electronic properties by strain [20], low-dimensional phosphorene derivatives [54], and new phosphorene polymorphs [55]. Extensive researches about device applications in gas sensors [56], electronics [57], and photovoltaic [58] have also been made. With so rich research results, the fundamental properties and applications have already been exposed; the focus has shifted from studying phosphorene itself to the various applications 


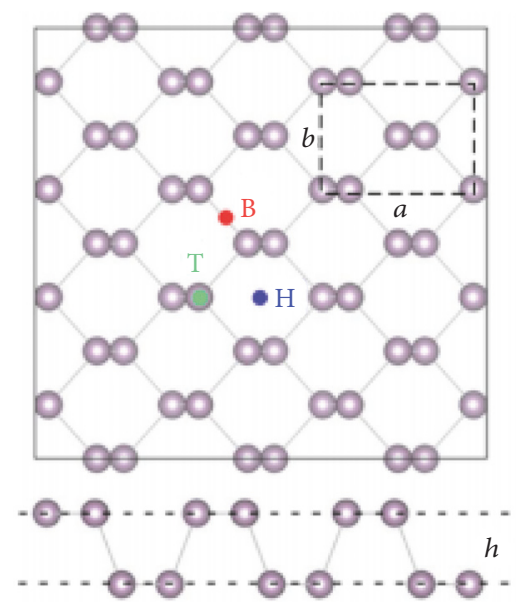

FIGURE 2: High-symmetry adsorption sites of adatoms [17].

TABLE 2: Favorable adsorption sites, binding energies $\left(E_{\mathrm{ad}}\right)$, and electronic and magnetic properties of adatoms on the phosphorene [17].

\begin{tabular}{lccccccc}
\hline & \multicolumn{7}{c}{ Adsorption on the black P sheet } \\
Atom & Site & $E_{\mathrm{ad}}(\mathrm{eV})$ & $h_{\mathrm{X}}(\AA)$ & $l_{\mathrm{X}-\mathrm{P}}(\AA)$ & $\delta_{\mathrm{P}}(\AA)$ & $M\left(\Delta E_{M}\right)$ & Properties \\
\hline $\mathrm{Li}$ & $\mathrm{H}$ & 1.93 & 1.43 & 2.48 & 0.12 & - & n-type doping \\
$\mathrm{Na}$ & $\mathrm{H}$ & 1.35 & 1.99 & 2.84 & 0.11 & - n-type doping \\
$\mathrm{Be}$ & $\mathrm{H}$ & 1.79 & 0.69 & 2.07 & 0.39 & - Defect states in gap \\
$\mathrm{Mg}$ & $\mathrm{H}$ & 0.66 & 1.78 & 2.70 & 0.26 & - Defect states in gap \\
\hline
\end{tabular}

and more complex phenomena based on phosphorene. It is expected that the prospects of phosphorene material and devices will be better.

This paper will discuss the structure and electronic and magnetic properties of phosphorene adsorbed with adatoms $[21,22,59]$, including alkali and alkaline-earth atoms [60], nonmetallic atoms, transition-metal atoms, and noble metal atoms [17]. The adsorption sites of adatom, as shown in Figure 2, can have the following four choices: $\mathrm{H}, \mathrm{B}, \mathrm{T}$, and $\mathrm{V}$ site; the hollow site $(\mathrm{H})$ is at the center of buckled hexagon, the bridge site (B) is at the midpoint of phosphorous-phosphorous (P-P) bond, the top site (T) is above the upper $\mathrm{P}$ atom, and the valley site $(\mathrm{V})$ is above the lower $\mathrm{P}$ atom [17]. We also discuss the possible applications of phosphorene adsorbed with adatoms in noble electronic, optoelectronic, and nanomechanical devices. Finally, we look forward to the future that the common adsorption, molecular adsorption, and the transport properties of phosphorene will be explored fully. The detailed study of adatom adsorption on phosphorene will be described then.

\section{Phosphorene with Adatoms}

2.1. Alkali and Alkaline-Earth Adatoms. In the first place, we research the interaction between $\mathrm{Li}, \mathrm{Na}, \mathrm{Be}$, and $\mathrm{Mg}$ adatoms and phosphorene, which is largely caused by the high binding energy, slight molecular distortion, and outstanding charge transfer for alkali and alkaline-earth adatoms; the optimized adsorption sites are all the $\mathrm{H}$ site, as shown in Figure 3.
In addition, Table 2 represents the binding energies $\left(E_{\mathrm{ad}}\right)$ and electronic and magnetic properties of adatoms on the phosphorene. The binging energies are 1.93, 1.79, 1.35, and $0.66 \mathrm{eV}$ for $\mathrm{Li}, \mathrm{Be}, \mathrm{Na}$, and $\mathrm{Mg}$, respectively, which appears in the order of $E_{\mathrm{ad}}(\mathrm{Li})>E_{\mathrm{ad}}(\mathrm{Be})>E_{\mathrm{ad}}(\mathrm{Na})>E_{\mathrm{ad}}(\mathrm{Mg})$. The bonding between $\mathrm{Li} / \mathrm{Be}$ and the phosphorene sheet is stronger than that of the $\mathrm{Na} / \mathrm{Mg}$ case, which is caused by the smaller adsorption heights and shorter bond lengths in the $\mathrm{Li} / \mathrm{Be}$ adsorption. It should be noticed that phosphorene adsorbed with $\mathrm{Li} / \mathrm{Be}$ adatoms is more stable and appropriate to be used in practical application.

2.1.1. Numerous Li Adatoms Case. Figure 4(a) shows that the charge transfer in the whole phosphorene system adsorbed with $\mathrm{Li}$ adatoms. The calculations of the charge transfer are obtained from $\Delta \rho=\rho_{\text {total }}-\rho_{\text {Psheet }}-\rho_{\text {Liatom }}$, where $\rho_{\text {total }}$ is total charge density of the Li adsorbed phosphorene sheet and $\rho_{\text {Psheet }}$ and $\rho_{\text {Liatom }}$ are charge density of pristine phosphorene and Li atom, respectively [17]. The obvious charge accumulation between the $\mathrm{Li}$ and $\mathrm{P}$ atoms manifests that the Li adsorption can form covalent bonding [17], which can make phosphorene adsorbed with $\mathrm{Li}$ adatoms promising anode materials for Li-ion batteries.

The case that numerous $\mathrm{Li}$ adatoms are adsorbed on the one side of phosphorene has been researched. There are two stable phases as displayed in Figures 4(b) and 4(c), namely, phase I and phase II. The $\mathrm{H}$ site has been examined as the most suitable site for both phases. And its total energy values of -25.267 and $-25.276 \mathrm{eV}$ per unit cell 


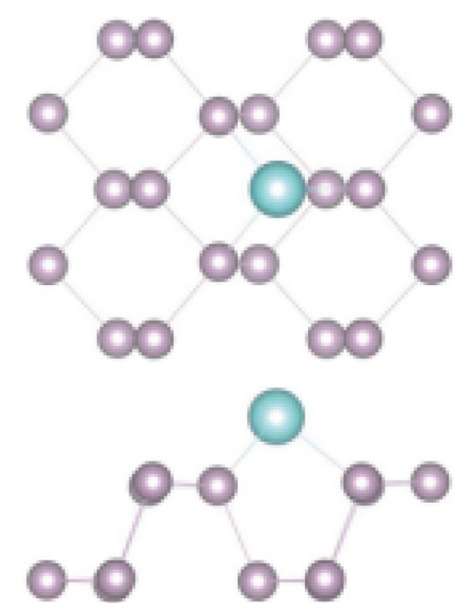

FIGURE 3: The typical adsorption structures of the alkali and alkaline-earth adatoms [17].

manifest that phase I is metastable. Moreover, the adsorption energies of $\mathrm{Li}$ adatoms of two stable phase are -1.61 and $-1.62 \mathrm{eV}$, respectively, which are lower than the cohesive energy $-1.60 \mathrm{eV}$ per Li atom of bulk lithium, indicating that two phases are more thermodynamically stable than the pure phosphorene and bulk lithium. The differences between two phases are significant, and the calculated lattice constants are 4.77 and $4.87 \AA$ along the $x$ direction and 3.23 and $3.24 \AA$ along the $y$ direction, respectively. It is interesting to notice that the upper phosphorene layer in phase I maintains being flat, which in phase II tilts clearly, which can be proved by a longer bond length between $\mathrm{Li}$ and $\mathrm{P}$ atoms in phaseI than that in phase II. The difference found is that phase II has a larger band gap $(0.4 \mathrm{eV})$ and sharp band edges around $-1.5 \mathrm{eV}$ below the Fermi levels, whereas phase I has a smaller band gap $(0.1 \mathrm{eV})$ and band tail states [18]. From the researches above, we can realize that phase II has a longer lattice constant so that Lil can have more space to get closer to $\mathrm{P}$ atoms and form more stronger bonding. Furthermore, we can conduct research about the condition that two Li atoms are adsorbed on the two sides of phosphorene in one unit cell, which can have complementary and contrast effects.

2.1.2. A Single Adatom Case. The case of a single adatom adsorption on the phosphorene sheet has also been investigated. In order to investigate the band gap of phosphorene adsorbed with alkali and alkaline-earth adatoms, the DOSs of them have been studied, which is shown in Figure 4(d). The adsorption of alkali and alkaline-earth adatoms can generate charge transfer, the $\mathrm{Li}$ atom donates $0.67 \mathrm{e}$ to the phosphorene, and the $\mathrm{Na} / \mathrm{Be} / \mathrm{Mg}$ atom loses charges ranging from 0.5 to $0.6 \mathrm{e}$, which affects the electronic property of phosphorene. The energy of $\mathrm{Li}$ adatom $\mathrm{s}$ orbitals and conduction band minimum (CBM) are -2.91 and $-4.14 \mathrm{eV}$, respectively. The high energy of s orbital makes Li adatom donate its electron to the conduction band, resulting in phosphorene being an $\mathrm{n}$ type semiconductor. The status of $\mathrm{Na}$ adatom is the same as the Li case; the Fermi level also moves into conduction bands, making the phosphorene sheet an n-type semiconductor, while, for the Be and $\mathrm{Mg}$ adatoms, due to the mid-gap defect states in the phosphorene [61], the band gaps are decreased to 0.18 and $0.4 \mathrm{eV}$, respectively [17]. The above studies are limited to the electronic property of phosphorene adsorbed with a single adatom; the mechanical and magnetic properties of the whole system need to be involved.

\subsection{Nonmetallic Adatoms}

2.2.1. Three Ways of Oxygen Atom Adsorption. Once nonmetallic adatoms $(\mathrm{O}, \mathrm{B}, \mathrm{C}$, and $\mathrm{N})$ are adsorbed on the phosphorene, the electronic properties of phosphorene will be changed [62]. In addition, these nonmetallic adatoms demonstrate a more strong adsorption than that of alkali and alkaline-earth metal adatoms. In the first place, we focus on oxygen $(\mathrm{O})$ adatoms adsorbed at three different sites [63-65]. The lowest energy but the highest binding energy structure is an $\mathrm{O}$ adatom adsorbed at the $\mathrm{T}$ site of phosphorene (Figure 5(a)(A)). The bond between a $\mathrm{O}$ atom and a $\mathrm{P}$ atom is short, about $1.5 \AA$. Moreover, the P-O bond aligns perpendicular to the surface with a slight tilt from the normal direction by $44.5^{\circ}$ [66]. And the binding energy is very high, $2.08 \mathrm{eV}$ at the PBE level. Such a high binding energy makes the process of $\mathrm{O}$ adatom adsorption exothermic strongly [67]. From Figure 5(a)(B), it is shown that there are no states in the gap so that the defect is electrically neutral. The DOS of the $\mathrm{O}$ adatom adsorption system is similar to that of pristine phosphorene.

The second lowest energy structure is an $\mathrm{O}$ adatom adsorption at the $\mathrm{V}$ site of phosphorene, as shown in Figure 5(b). The $\mathrm{O}$ adatom is deep inside the lattice, forming a bridge between two $\mathrm{P}$ atoms and making its adjacent $\mathrm{P}$ atom outside the phosphorene surface [68]. Researches show that P-O bond lengths are $1.66 \AA$ and $1.68 \AA$ and the P-O-P bond angle is $129.6^{\circ}$. Compared with pristine phosphorene $(2.11 \AA)$, the thickness is $2.87 \AA$ now. Such a huge change of the structure makes no difference, due to the fact that binding energy is $1.66 \mathrm{eV}$ at the PBE level and the formation of bridges is highly exothermic. Similar to the case of $\mathrm{O}$ adatoms adsorbed at the $\mathrm{T}$ site, the defect is also electrically 


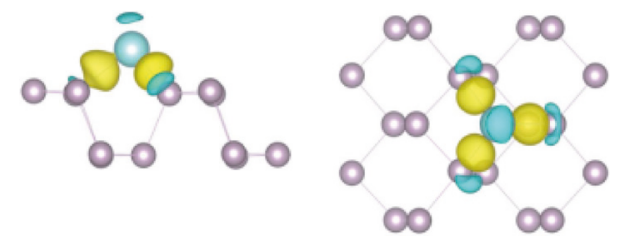

(a) Li@H/black P

(b) Phase I of $\mathrm{P}_{4} \mathrm{Li}_{2}$

(c) Phase II of $\mathrm{P}_{4} \mathrm{Li}_{2}$
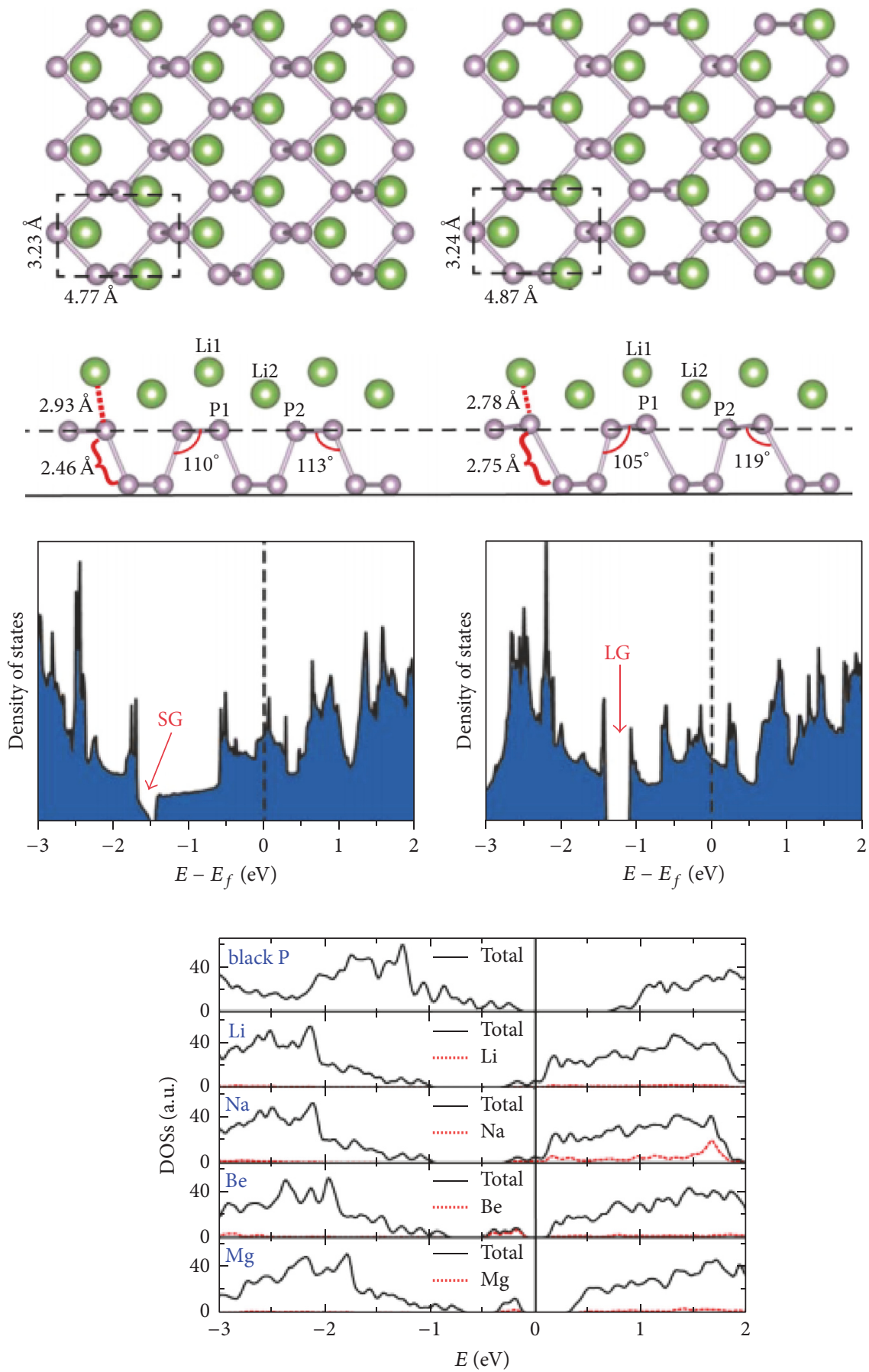

(d)

FIGURE 4: (a) Charge densities of Li adatoms on phosphorene [17]. (b) Phase I [18] and (c) phase II of Li doped phosphorene, the difference at $-1.5 \mathrm{eV}$ below the Fermi level: phase I possesses a smaller gap (SG) whereas phase II has a larger gap (LG) [18]. (d) DOSs of phosphorene adsorbed with Li-Mg adatoms [17]. 


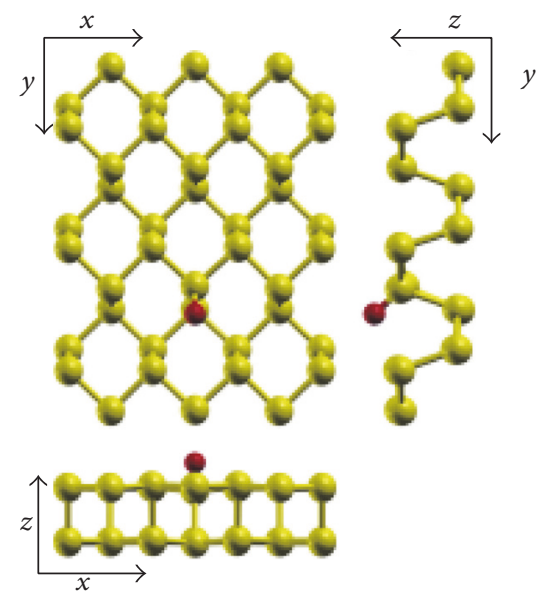

(A)

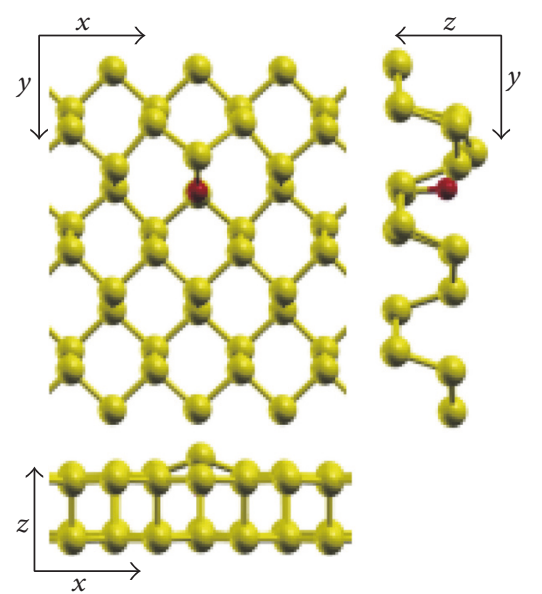

(A)

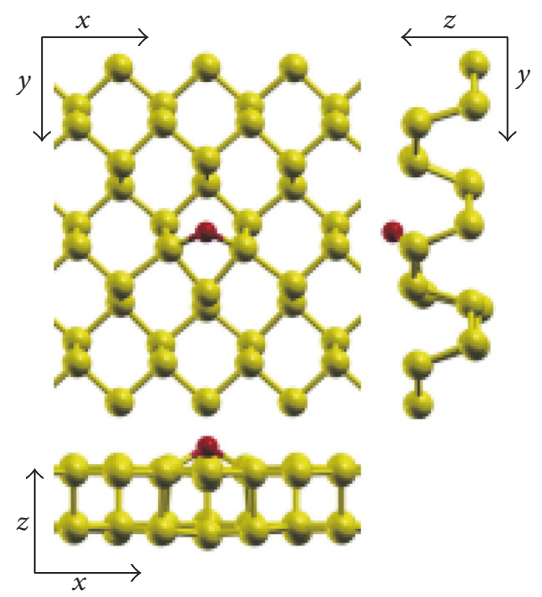

(A)
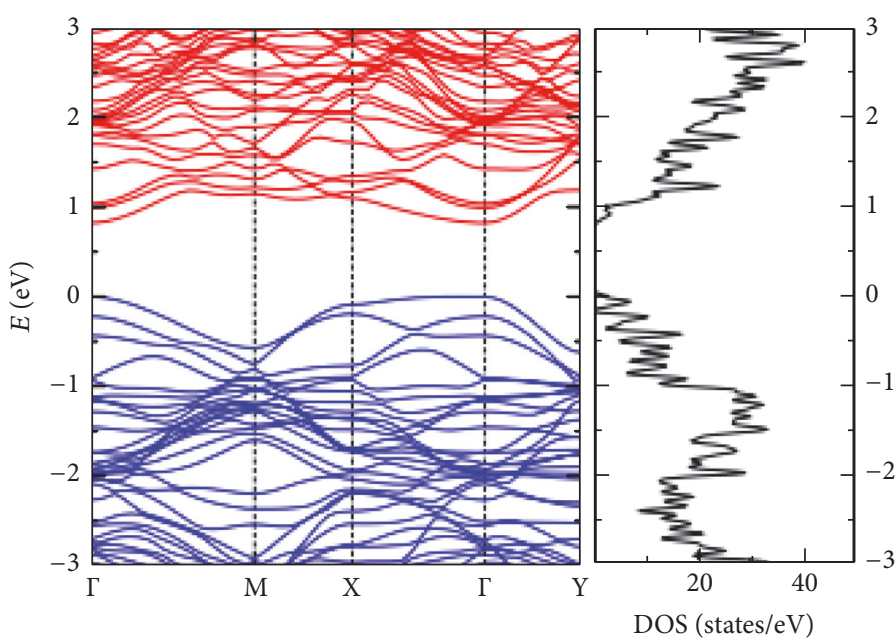

(B)

(a)
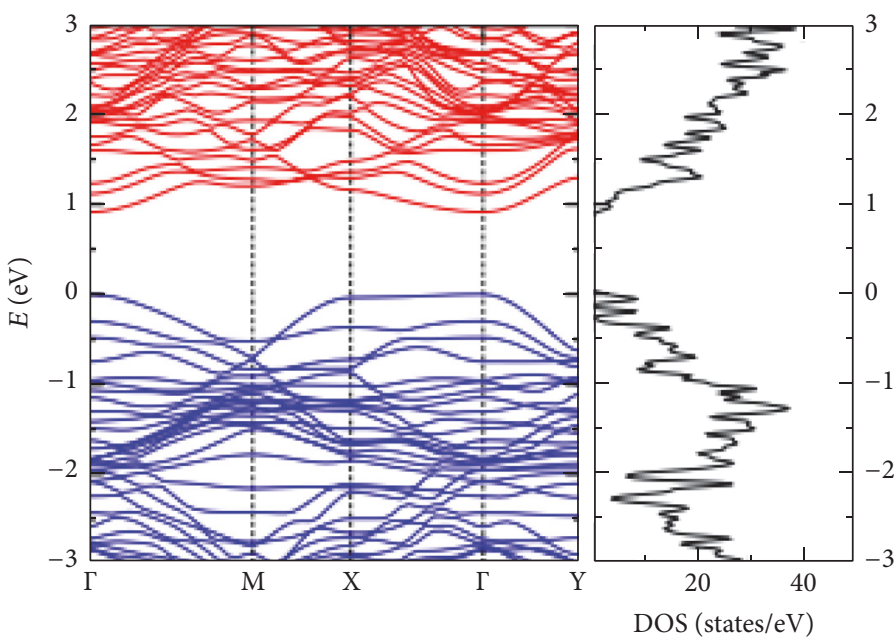

(B)

(b)

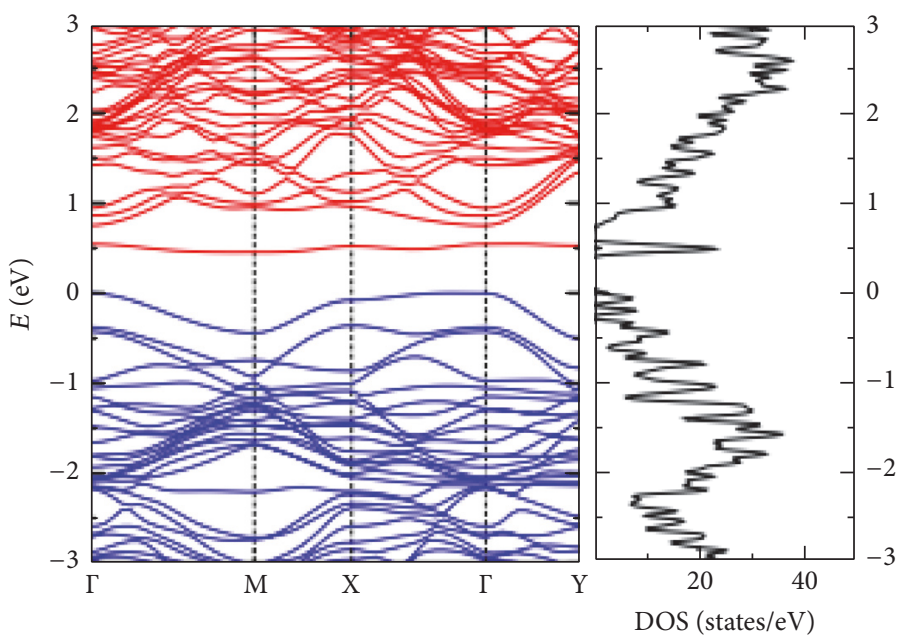

(B)

(c)

Figure 5: $\mathrm{O}$ adatoms adsorbed at the (a) T, (b) V, and (c) $\mathrm{H}$ site of phosphorene: (A) the structure of $\mathrm{O}$ adatoms adsorbed at the T, V, and $\mathrm{H}$ site of phosphorene, respectively. (B) The electronic band structure (left) and DOS (right) [14]. 


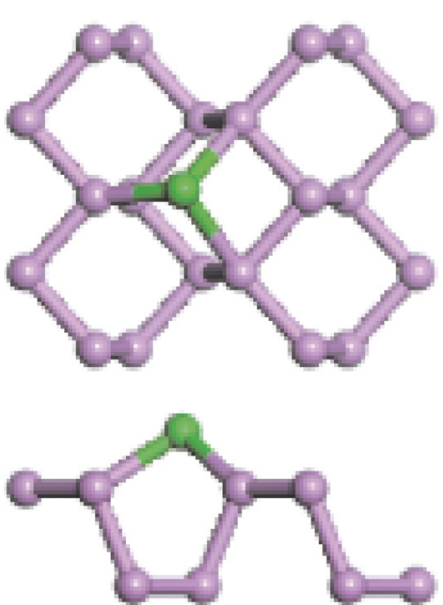

(a) B@H
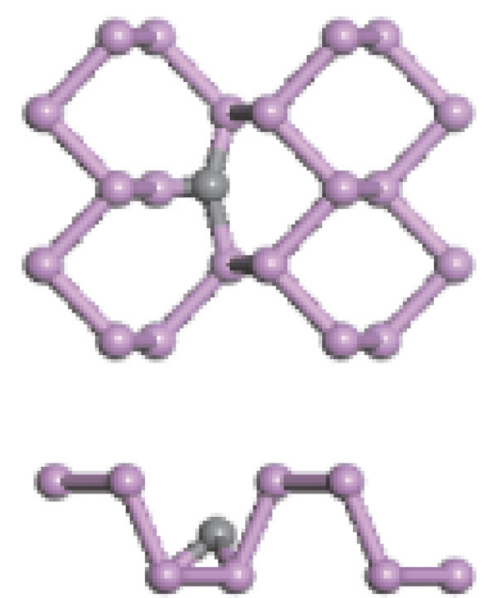

(b) C@E

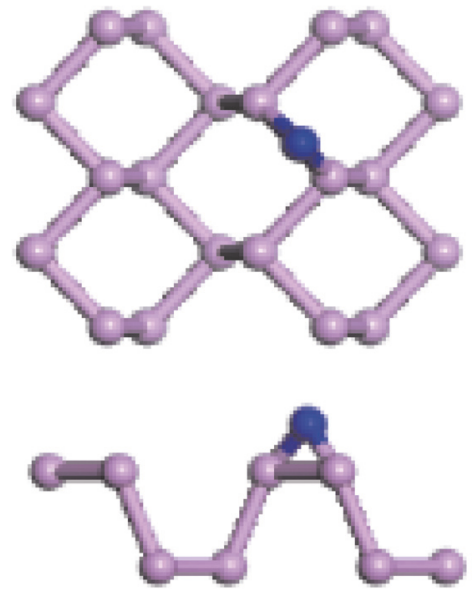

(c) $\mathrm{N} @ B$

FIGURE 6: Optimized adsorption sites of B-O atoms; X@Y represents that the $\mathrm{X}$ atom is at the $\mathrm{Y}$ site of phosphorene [17].

neutral without states forming in the middle of the gap (Figure 5(b)(B)).

The last possible structure has been studied out: the $\mathrm{O}$ adatom is adsorbed on the top of phosphorene along the zigzag direction, connecting two $\mathrm{P}$ atoms with a horizontal bridge, which is demonstrated in Figure 5(c)(A). The P$\mathrm{O}$ bonds are now $1.75 \AA$ and the angle is $107.9^{\circ}$. From Figure 5(c)(B), The horizontal bridge defect produces a midgap state near the conduction band, which is formed by the $\mathrm{p}_{x}$ orbital of the $\mathrm{O}$ adatom and the $\mathrm{p}$ orbitals of the two $\mathrm{P}$ atoms in the $\mathrm{P}-\mathrm{O}$ system. Otherwise, the valence band state is disturbed, which can be used to generate recombination lines in luminescence experiments [19, 23-25, 69].

The detailed studies about $\mathrm{O}$ adatoms adsorption on phosphorene provide a deep understanding of oxygen defects in phosphorene, which may prevent forming more complex defects and deterioration when phosphorene is exposed to air.

\subsubsection{Other Nonmetallic Adatoms Adsorption. Other non-} metallic adatom cases are summarized next. The electronic variation of phosphorene with nonmetallic adatoms is mainly controlled by the electronegativity of the nonmetallic atom. The electronegativities of $\mathrm{B}$ and $\mathrm{C}$ adatoms are equal to that of $\mathrm{P}$ atom nearly; they tend to form $\mathrm{sp}^{2}$ bonds with $\mathrm{P}$ atom, whereas the $\mathrm{N}$ adatom has more electronegativity than $\mathrm{P}$ atom. For the B adatom shown in Figure 6(a), its ideal adsorption site is $\mathrm{H}$ site, and the binding energy is $3.58 \mathrm{eV}$. Figure 6(b) represents that the $\mathrm{C}$ adatom tends to the $\mathrm{B}$ site with a binding energy of $5.11 \mathrm{eV}$. With the lowest binding energy of $3.49 \mathrm{eV}$ among the four adatoms, $\mathrm{N}$ adatoms prefer the $\mathrm{B}$ site. When the $\mathrm{C}$ adatom has stabilized, we can find that $\mathrm{C}$ adatom inserts the upper $\mathrm{P}$ atoms without a buckling height. Furthermore, the $\mathrm{C}$ adatom makes the three connecting $\mathrm{P}$ atoms more strong. While the $\mathrm{N}$ adatom remains the previous site with a buckling height of $0.61 \AA$, which can be seen in Figure 6(c), when the $\mathrm{N}$ adatom adsorbs at the bridge site, the P-P bond is replaced by P-N-P connection.
Then the DOSs of phosphorene adsorbed with B, C, and $\mathrm{N}$ adatoms will be studied, which is shown in Figure 7. The $\mathrm{B}$ adatom induces a spin-polarization with a magnetic moment of $0.95 \mu \mathrm{B}$, which is mainly originated from the $\mathrm{P}$ atoms in the surrounding of the $\mathrm{B}$ atom. In addition, the energy gained from the spin-polarization, called magnetic energy, is only $1 \mathrm{meV}$, denoting that the magnetism of $\mathrm{B}$ adsorbed phosphorene is hidden by the thermal fluctuation of the ambient environment. From the DOSs of B adatoms, it can be confirmed that there is a defect state crossing the Fermi level so that phosphorene adsorbed with $\mathrm{B}$ adatom becomes metallic. For the $\mathrm{C}$ adatom, the unoccupied defect state of C $2 p$ orbitals causes the gap decrease to $0.26 \mathrm{eV}[61,68]$. As for the $\mathrm{N}$ adatom, the Fermi level decreases due to the remarkable peak on the valence band maximum (VBM) [60], which makes the phosphorene sheet a p-type semiconductor. Finally, we focus on the $\mathrm{O}$ adatom adsorption at the $\mathrm{T}$ site of phosphorene, which is discussed above [26]. Surface adsorption with nonmetallic adatoms brings about so rich electronic and magnetic properties, which provides a theoretical basis of applications in nanoelectronics and spintronics.

2.3. Noble Metal Adatoms. In this section, we study the adsorption of noble metal adatoms $(\mathrm{Ag}, \mathrm{Au}$, and $\mathrm{Pt})$. As for noble metal adatoms, the most appropriate adsorption site is all $\mathrm{H}$ site, and the binding energies are 1.14, 1.61, and $4.82 \mathrm{eV}$ for $\mathrm{Ag}, \mathrm{Au}$, and $\mathrm{Pt}$, respectively. Among the three adatoms we investigated, the binding energy of Pt is the highest while $\mathrm{Ag}$ is the least. There has been published work demonstrating that the power of bonding and reactivity of a noble metal atom lie in whether its d-band is filled fully or not and the condition of its d-band crossing the Fermi level. The Pt adatom not only bonds with phosphorene strongly but also is reactive in phosphorene due to the fact that $\mathrm{d}$-band of $\mathrm{Pt}$ adatom is filled partly and throughout the Fermi level. On the other band, owing to the fact that d-band is filled fully 


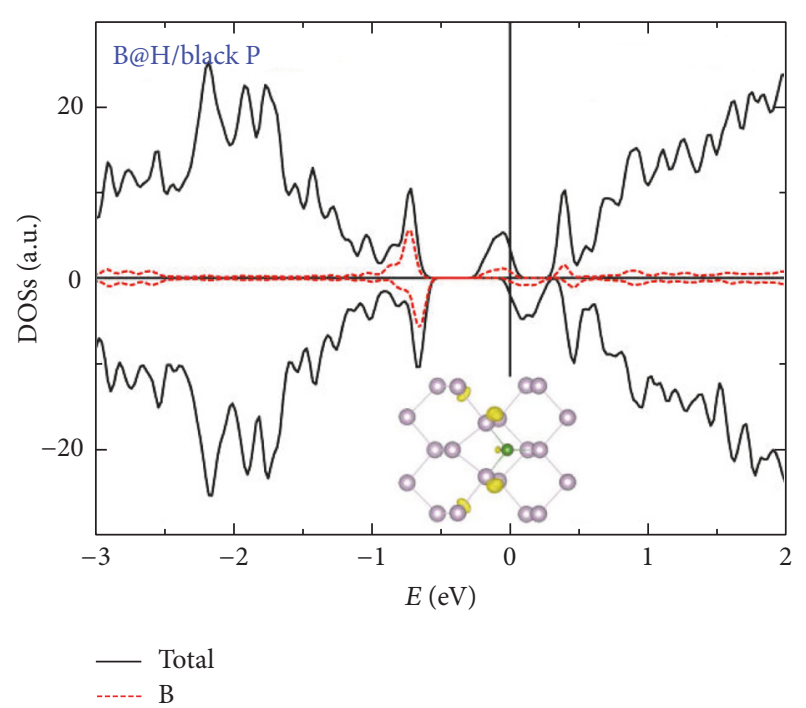

(a)

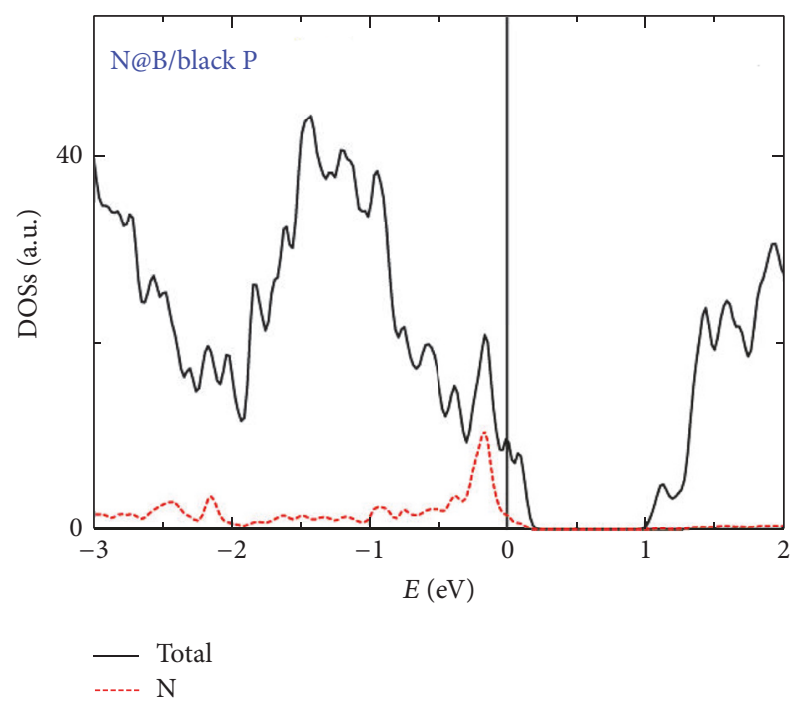

(c)

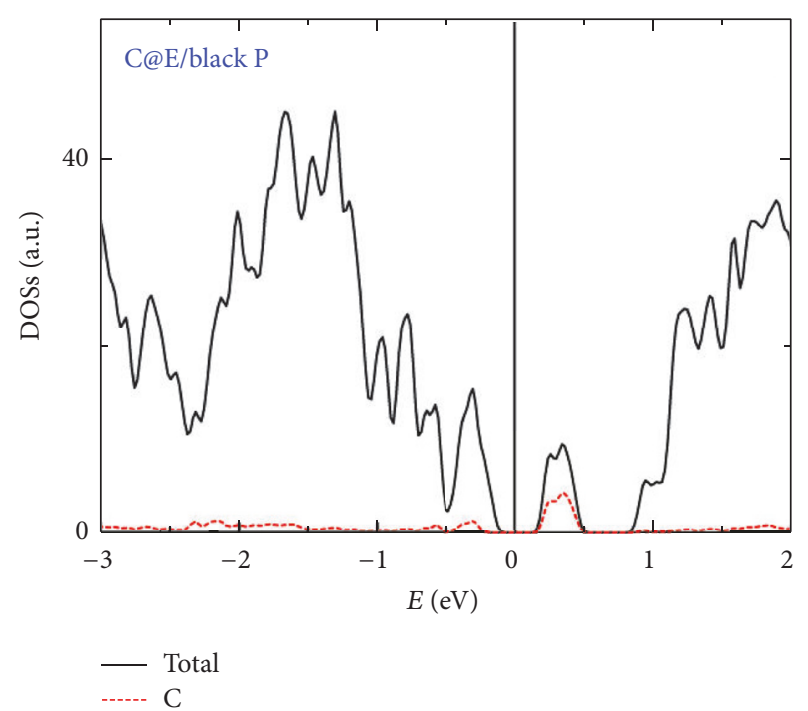

(b)

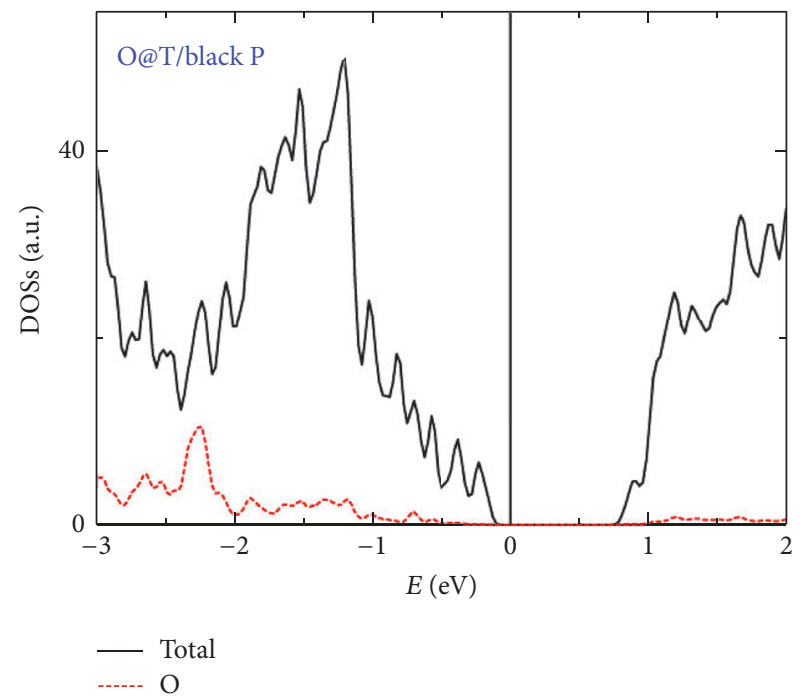

(d)

FIgURE 7: DOSs of phosphorene adsorbed with (a) B, (b) C, (c) N, and (d) O adatoms [17].

and does not cross the Fermi level, the reactivity of $\mathrm{Ag}$ and $\mathrm{Au}$ adatoms is feeble. These findings account for the different results of $\mathrm{Ag}, \mathrm{Au}$, and $\mathrm{Pt}$ adsorption.

The DOSs of the phosphorene adsorbed with noble metal adatoms can be found in Figure 8. For Ag atom, a result is that phosphorene with $\mathrm{Ag}$ is an n-type semiconductor, due to the fact that Ag adatom upshifts the Fermi level into the conduction bands.

Compared with Ag adatom, Au possesses a more steady magnetism, so phosphorene adsorbed with $\mathrm{Au}$ adatoms has a magnetism of $1 \mu \mathrm{B}$ [15]. It is emerged in the DOSs that the neighborhood of the Fermi level occupies spin-polarized defect states, which makes phosphorene a bipolar spingapless semiconductor.

For the Pt atom, its defect states are primarily seated in the valence bands, so the electronic structure of phosphorene is affected slightly by adsorbing with Pt atoms [16].
2.4. Transition-Metal Adatoms. Next, the adsorption of transition-metal adatoms ( $\mathrm{Co}, \mathrm{Fe}, \mathrm{Ni}, \mathrm{Cu}$, and $\mathrm{Zn}$ ) will be discussed [21]. We find that the $\mathrm{Zn}$ atom is not appropriate to decorate the phosphorene; it can be stated that the optimized adsorption site is $\mathrm{H}$ site with a large distance of $2.39 \AA$ above $\mathrm{P}$ atoms and the binding energy is only $0.15 \mathrm{eV}$, which can lead phosphorene to be disturbed by the surrounding. However, from the binding energy of $\mathrm{Fe}, \mathrm{Co}, \mathrm{Ni}$, and $\mathrm{Cu}(\mathrm{Fe}: 2.98 \mathrm{eV}$, Co: $3.75 \mathrm{eV}, \mathrm{Ni}: 4.41 \mathrm{eV}, \mathrm{Cu}: 2.15 \mathrm{eV}$ ), we can detect that the adsorption of $\mathrm{Fe}, \mathrm{Co}, \mathrm{Ni}$, and $\mathrm{Cu}$ on the phosphorene is powerful. From the results we can make the conclusion that phosphorene adsorbed with $\mathrm{Fe}, \mathrm{Co}, \mathrm{Ni}$, and $\mathrm{Cu}$ holds an absolute advantage.

The stability and electronic properties of Co adatom adsorption on the phosphorene are studied firstly. Figure 9 shows that the adsorption with Co adatoms leads to slight lattice distortion [21] and the Co adatom connects $\mathrm{P}$ atoms 

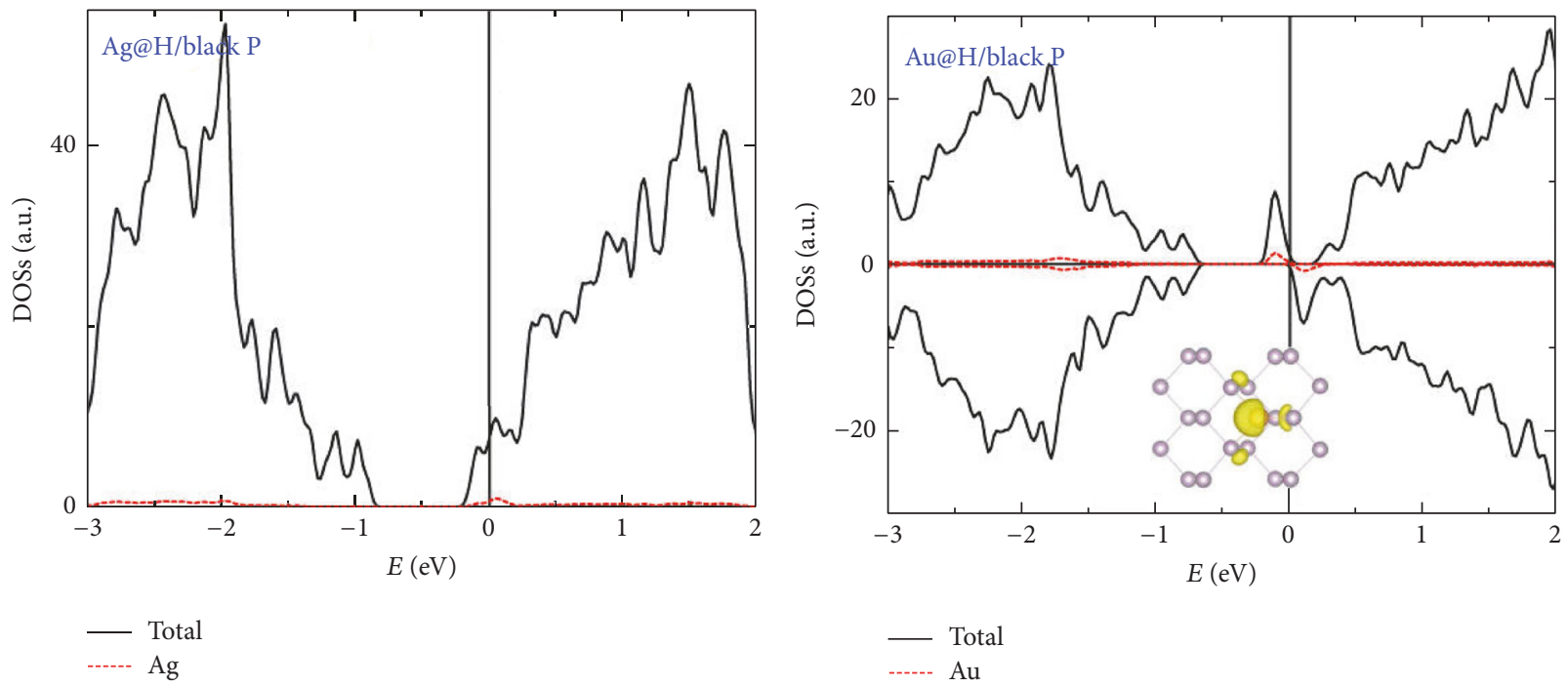

(a)

(b)

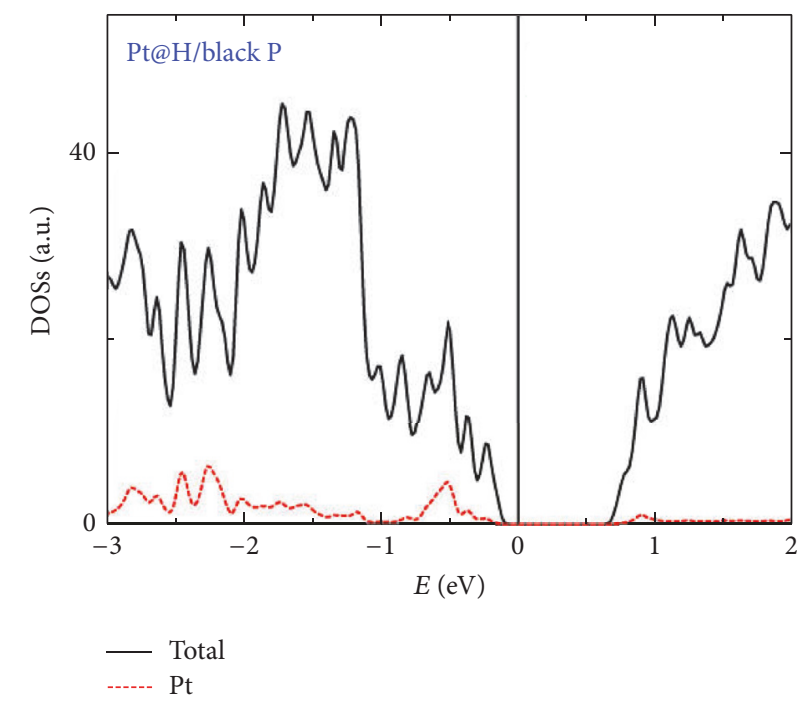

(c)

FIGURE 8: DOSs of phosphorene adsorbed with (a) Ag, (c) Au, and (e) Pt adatoms [17].

with three bonds in two zigzag ridges. The adsorption energy of Co adatoms is the second highest among transitionmetal adatoms. Researches present that the concentration of Co adatoms is about $1.23 \%$. In addition, the coadsorbed phosphorene has a total magnetic moment of $1 \mu \mathrm{B}$. We can increase the concentration ratio of Co adatoms; the magnetic moment may increase or disappear. We can also connect one Co adatom with three $\mathrm{P}$ atoms in two armchair ridges; the lattice may become more stable. There are so many investigations remaining to be conducted so that we can go deep into the study above.

The discussion of DOSs of these transition-metal adatoms is presented in Figure 10. Firstly, the band gap of phosphorene adsorbed with $\mathrm{Fe}$ is reduced to $0.22 \mathrm{eV}$ with some defect states in the band gap [14]. For the Co adatom, the valence maximum and the conduction minimum have different spins, but they are connected to each other at the Fermi level, so the phosphorene becomes a bipolar spin-gapless semiconductor [59]. For the Ni adatom, the valence maximum has so many defect states that the band gap decreases. With regard to the $\mathrm{Cu}$ adatom, the phosphorene sheet becomes an n-type semiconductor due to the fact that $\mathrm{Cu}$ adatom moves the Fermi level upward [26]. It has been reported that there are covalent interaction between Fe adatom and the next $\mathrm{P}$ atom and a compound of covalent and ionic interactions for $\mathrm{Co}, \mathrm{Ni}$, and $\mathrm{Cu}$ adsorption. Furthermore, the addition of transitionmetal atoms changes phosphorene into a semiconductor, such that the Co- and $\mathrm{Cu}$-adsorbed systems maintain the property of semiconductor. Transition-metal atoms, such as $\mathrm{Fe}$ and $\mathrm{Ni}$ atom, can also decrease the band gap, which is mainly because the spin-polarized electrons of adatoms shift 


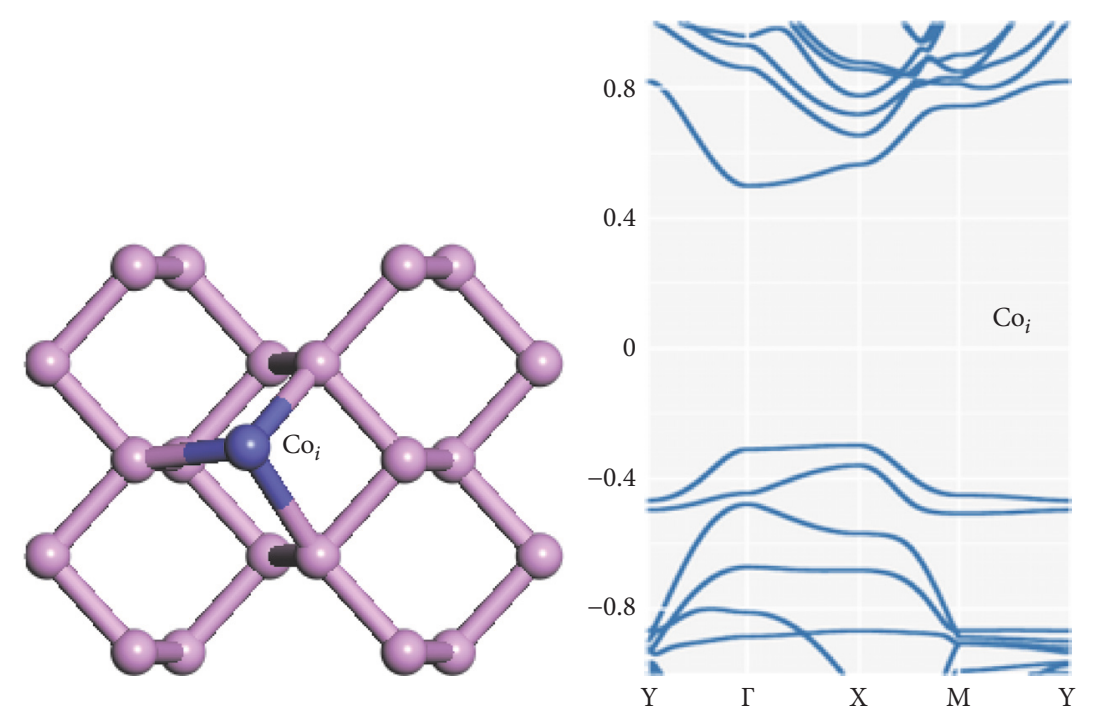

FiguRE 9: Ball-and-stick representation of phosphorene with adsorbed Co defect; electronic band structures for coadsorbed phosphorene [4].

to the non-spin-polarized phosphorene and the 4 s electrons of transition-metal atoms rise to the $3 \mathrm{~d}$ orbitals.

It is verified that phosphorene is a layer-dependent $2 \mathrm{D}$ material; the structure and electronic and magnetic properties of transition-metal adatoms on bilayer and few layers phosphorene have not been involved. Moreover, the topics of phosphorene coadsorbed with transition-metal adatoms and other types of atoms have not been focused on.

\section{Conclusions}

Phosphorene, owing to its remarkable features, such as a large direct band gap of $1.5 \mathrm{eV}$, a high on/off ratio of over $10^{4}$, and a high carrier mobility of up to $10000 \mathrm{~cm}^{2} \mathrm{~s}^{-1} \mathrm{v}^{-1}$, has become a new important $2 \mathrm{D}$ material. As we all know, the way of adsorbing atoms on the phosphorene can change the structure and electronic and magnetic properties of phosphorene, which provides a wider application in transistors, optoelectronics, batteries, and gas storage [28, 29]. These adatoms are alkali and alkaline-earth atoms, nonmetallic atoms, noble metal atoms, and transition-metal atoms:

(1) For alkali and alkaline-earth adatoms, their favourite sites are $\mathrm{H}$ site. Moreover, phosphorene decorated with $\mathrm{Li}$ and $\mathrm{Na}$ adatoms becomes an n-type semiconductor, and $\mathrm{Be}$ and $\mathrm{Mg}$ adatoms make the band gap of phosphorene decrease to 0.18 and $0.14 \mathrm{eV}$, respectively.

(2) Nonmetallic adatoms include B, C, N, and O: B adatoms can induce the property of metal. $\mathrm{C}$ adatoms cause the band gap decrease to $0.26 \mathrm{eV}$. And $\mathrm{N}$ adatoms make the phosphorene a p-type semiconductor [16]. Some small peaks in the valence band show that $\mathrm{O}$ adatoms can affect the electronic property of phosphorene sheet slightly.

(3) As for noble metal atoms ( $\mathrm{Ag}, \mathrm{Au}$, and $\mathrm{Pt}$ ), the most appropriate adsorption sites are all $\mathrm{H}$ sites.
Phosphorene with $\mathrm{Ag}$ is an n-type semiconductor, and phosphorene adsorbed with $\mathrm{Au}$ atoms has a magnetism of $1 \mu \mathrm{B}$. For the $\mathrm{Pt}$ atom, the electronic property of phosphorene is changed.

(4) Referring to transition-metal adatoms, Fe and Ni's band gap is reduced with some defect state. Adsorbed with Co adatoms, the phosphorene becomes a polar spin-gapless semiconductor, while the phosphorene with $\mathrm{Cu}$ becomes an n-type semiconductor [30]. But $\mathrm{Zn}$ is not appropriate to adsorb on the phosphorene.

\section{Prospects}

Phosphorene has become a hot spot of two-dimensional nanomaterials research. The adsorption method can change the properties of phosphorene so largely that abundant researches have been carried out to find possible applications in noble electronic, optoelectronic, and nanomechanical devices [31]. For instance, the ways in which hydrogen [32] and oxygen adsorb on phosphorene have explained the surface reactivity and its oxidation behaviour. The alkali and alkaline-earth adatom adsorption on phosphorene provides the possibility of producing high density electronic sheet and battery-like applications $[26,33] \mathrm{Li}$ and $\mathrm{Na}$ adsorption of phosphorene are also applied to devise Li-ion [34-36] and $\mathrm{Na}$-ion batteries. Hybrid phosphorene that is created by noble metal adatoms provides great interest to researchers in catalysis and nanoelectronic devices [37, 38]. Besides, phosphorene decorated with transition-metal atoms can be applied for a plasmonic photocatalyst for hydrogen production from water-splitting and spintronic applications [7].

This article is mainly a summary of the single adatom adsorption on the phosphorene. In the future, the focus will be on the common adsorption and molecular adsorption [3942]. In a word, there are still a large number of difficulties about phosphorene remaining to be solved, such as the 


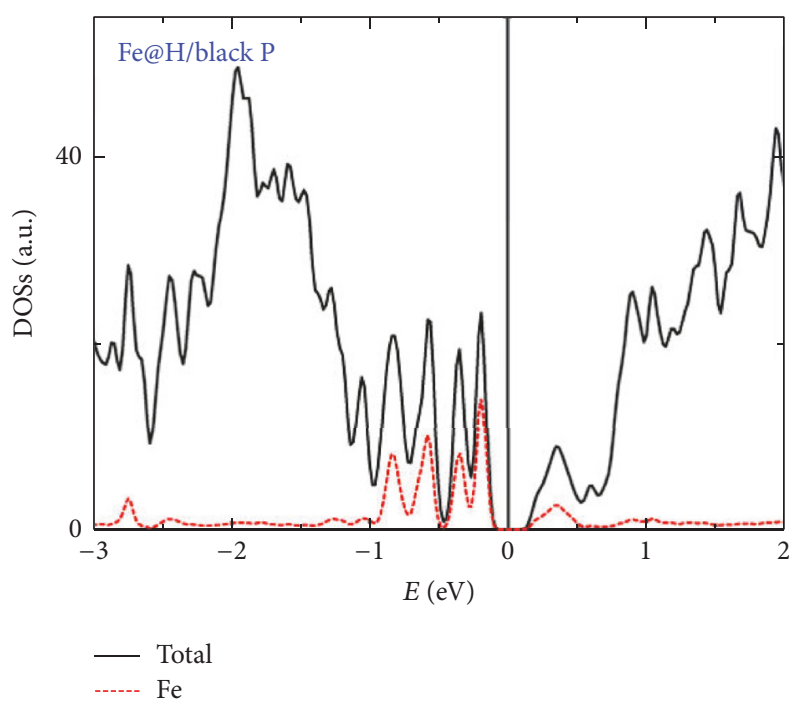

(a)

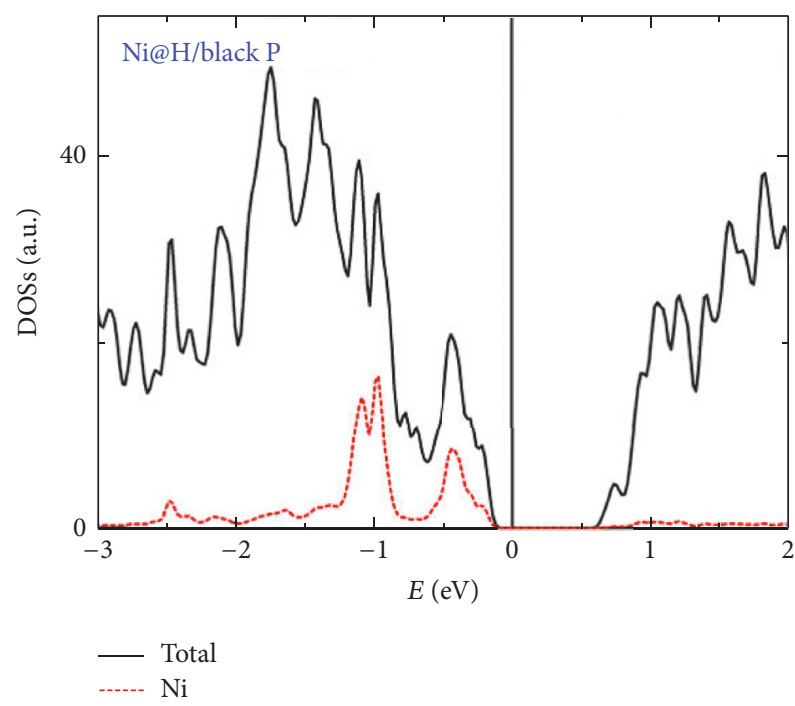

(c)

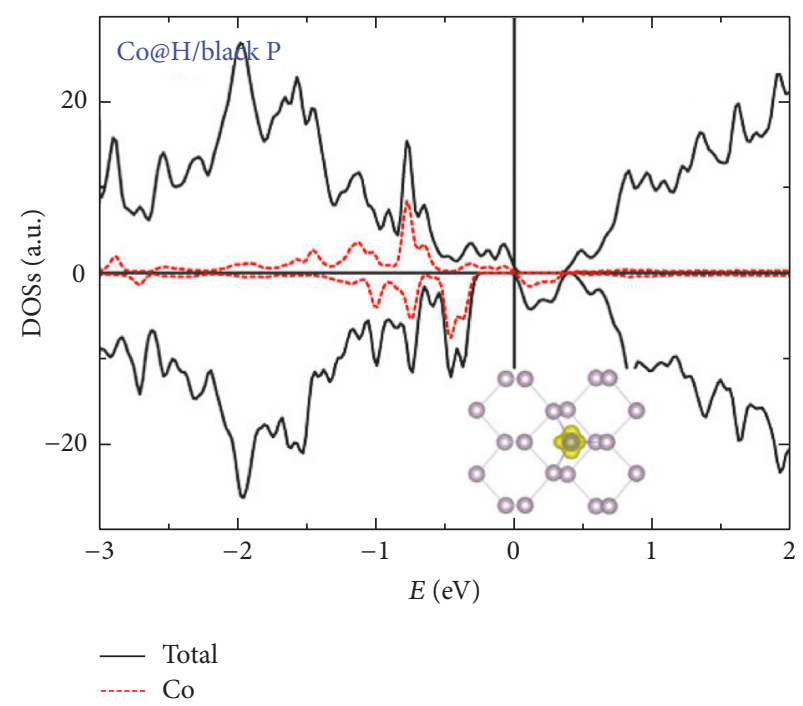

(b)

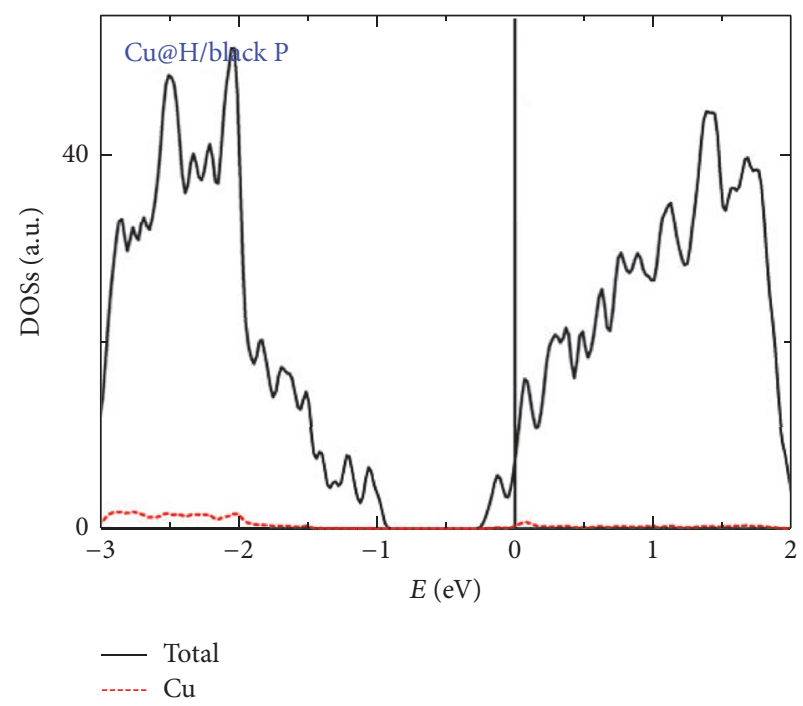

(d)

FIGURE 10: DOSs of phosphorene adsorbed with (a) Fe, (b) Co, (c) Ni, and (d) Cu adatoms [17].

transport properties of phosphorene $[43,44]$ and the application of real materials. The study of the adsorption of phosphorene is a small branch; it is believed that the exploration of phosphorene will become a raging fire.

\section{Conflicts of Interest}

The authors declare that they have no conflicts of interest.

\section{Acknowledgments}

This paper is supported by the Natural Science Foundation of China (Grant no. 11564008), the Scientific Research Foundation of Guilin University of Technology, and the Shanghai Supercomputer Center.

\section{References}

[1] A. K. Geim and K. S. Novoselov, "The rise of graphene," Nature Materials, vol. 6, no. 3, pp. 183-191, 2007.

[2] H. Wang, H. Yuan, S. Sae Hong, Y. Li, and Y. Cui, "ChemInform abstract: physical and chemical tuning of two-dimensional transition metal dichalcogenides," ChemInform, vol. 44, no. 9, article 2664, pp. no-no, 2014.

[3] T.-D. Chou, T.-W. Lee, S.-L. Chen et al., "The management of white phosphorus burns," Burns, vol. 27, no. 5, pp. 492-497, 2001.

[4] L. Li, Y. Yu, G. J. Ye et al., "Black phosphorus field-effect transistors," Nature Nanotechnology, vol. 9, no. 5, pp. 372-377, 2014.

[5] X. Ling, H. Wang, S. Huang, F. Xia, and M. S. Dresselhaus, "The renaissance of black phosphorus," Proceedings of the National Academy of Sciences of the United States of America, vol. 112, no. 15, pp. 4523-4530, 2015. 
[6] L. Seixas, A. Carvalho, and A. H. Castro Neto, "Atomically thin dilute magnetism in Co-doped phosphorene," Physical Review B, vol. 91, no. 15, Article ID 155138, 2015.

[7] H. Liu, Y. Du, Y. Deng, and P. D. Ye, "Semiconducting black phosphorus: synthesis, transport properties and electronic applications," Chemical Society Reviews, vol. 44, no. 9, pp. 2732 2743, 2015.

[8] S. Appalakondaiah, G. Vaitheeswaran, S. Lebègue, N. E. Christensen, and A. Svane, "Effect of van der Waals interactions on the structural and elastic properties of black phosphorus," Physical Review B-Condensed Matter and Materials Physics, vol. 86, no. 3, pp. 41-48, 2012.

[9] H. Liu, A. T. Neal, Z. Zhu et al., "Phosphorene: an unexplored 2D semiconductor with a high hole mobility," ACS Nano, vol. 8 , no. 4, pp. 4033-4041, 2014.

[10] T. Zhao, C. Y. He, S. Y. Ma et al., "A new phase of phosphorus: the missed tricycle type red phosphorene," Journal of Physics Condensed Matter, vol. 27, no. 26, Article ID 265301, 2015.

[11] M. Z. Rahman, C. W. Kwong, K. Davey, and S. Z. Qiao, "2D phosphorene as a water splitting photocatalyst: fundamentals to applications," Energy and Environmental Science, vol. 9, no. 3, pp. 709-728, 2016.

[12] J. Zhao, H. Liu, Z. Yu et al., "Rise of silicene: a competitive 2D material," Progress in Materials Science, vol. 83, pp. 24-151, 2016.

[13] S. Zhang, J. Yang, R. Xu et al., "Extraordinary photoluminescence and strong temperature/angle-dependent raman responses in few-layer phosphorene," ACS Nano, vol. 8, no. 9, pp. 9590-9596, 2014.

[14] X. Han, H. Morgan Stewart, S. A. Shevlin, C. R. A. Catlow, and Z. X. Guo, "Strain and orientation modulated bandgaps and effective masses of phosphorene nanoribbons," Nano Letters, vol. 14, no. 8, pp. 4607-4614, 2014.

[15] B. Sa, Y.-L. Li, J. Qi, R. Ahuja, and Z. Sun, "Strain engineering for phosphorene: the potential application as a photocatalyst," Journal of Physical Chemistry C, vol. 118, no. 46, pp. 2656026568, 2014.

[16] X. Peng, Q. Wei, and A. Copple, "Strain-engineered direct-indirect band gap transition and its mechanism in two-dimensional phosphorene," Physical Review B, vol. 90, no. 8, Article ID 085402, 2014.

[17] Y. Ding and Y. Wang, "Structural, electronic, and magnetic properties of adatom adsorptions on black and blue phosphorene: a first-principles study," Journal of Physical Chemistry C, vol. 119, no. 19, pp. 10610-10622, 2015.

[18] J. Deng, Z. Chang, T. Zhao, X. Ding, J. Sun, and J. Z. Liu, "Electric field induced reversible phase transition in Li doped phosphorene: shape memory effect and superelasticity," Journal of the American Chemical Society, vol. 138, no. 14, pp. 4772-4778, 2016.

[19] M. Batmunkh, M. Bat-Erdene, and J. G. Shapter, "Phosphorene and phosphorene-based materials-prospects for future applications," Advanced Materials, vol. 28, no. 39, pp. 8586-8617, 2016.

[20] A. S. Rodin, A. Carvalho, and A. H. Castro Neto, "Straininduced gap modification in black phosphorus," Physical Review Letters, vol. 112, no. 17, Article ID 176801, 2014.

[21] J. Sivek, H. Sahin, B. Partoens, and F. M. Peeters, "Adsorption and absorption of boron, nitrogen, aluminum, and phosphorus on silicene: Stability and electronic and phonon properties," Physical Review B-Condensed Matter and Materials Physics, vol. 87, no. 8, Article ID 085444, 2013.
[22] S.-S. Li, C.-W. Zhang, W.-X. Ji et al., "Tunable electronic and magnetic properties in germanene by alkali, alkaline-earth, group III and 3d transition metal atom adsorption," Physical Chemistry Chemical Physics, vol. 16, no. 30, pp. 15968-15978, 2014.

[23] K. L. Kuntz, R. A. Wells, J. Hu, and et al, "Control of surface and edge oxidation on phosphorene," Acs Applied Materials \& Interfaces, vol. 9, no. 10, article 9126, 2017.

[24] S. Nahas, B. Ghosh, S. Bhowmick, and A. Agarwal, "Firstprinciples cluster expansion study of functionalization of black phosphorene via fluorination and oxidation," Physical Review B-Condensed Matter and Materials Physics, vol. 93, no. 16, Article ID 165413, 2016.

[25] O. I. Malyi, K. V. Sopiha, C. Draxl, and C. Persson, "Stability and electronic properties of phosphorene oxides: from 0-dimensional to amorphous 2-dimensional structures," Nanoscale, vol. 9, no. 7, pp. 2428-2435, 2017.

[26] V. V. Kulish, O. I. Malyi, C. Persson, and P. Wu, "Adsorption of metal adatoms on single-layer phosphorene," Physical Chemistry Chemical Physics, vol. 17, no. 2, pp. 992-1000, 2015.

[27] L.-J. Kong, G.-H. Liu, and Y.-J. Zhang, "Tuning the electronic and optical properties of phosphorene by transition-metal and nonmetallic atom co-doping," RSC Advances, vol. 6, no. 13, pp. 10919-10929, 2016.

[28] P. Srivastava, K. P. S. S. Hembram, H. Mizuseki, K.-R. Lee, S. S. Han, and S. Kim, "Tuning the electronic and magnetic properties of phosphorene by vacancies and adatoms," Journal of Physical Chemistry C, vol. 119, no. 12, pp. 6530-6538, 2015.

[29] P. Kumar, B. S. Bhadoria, S. Kumar, S. Bhowmick, Y. S. Chauhan, and A. Agarwal, "Thickness and electric-field-dependent polarizability and dielectric constant in phosphorene," Physical Review B, vol. 93, no. 19, Article ID 195428, 2016.

[30] M. Lalitha, Y. Nataraj, and S. Lakshmipathi, "Calcium decorated and doped phosphorene for gas adsorption," Applied Surface Science, vol. 377, pp. 311-323, 2016.

[31] Y. Zhang, H. Wang, Z. Luo et al., "Lithium storage: an airstable densely packed phosphorene-graphene composite toward advanced lithium storage properties (Adv. Energy Mater. 12/2016)," Advanced Energy Materials, vol. 6, no. 12, Article ID 1600453, 2016.

[32] H. Xiao, X. Shi, and F. Hao, Development of a Transferable Reactive Force Field for Phosphorus-Hydrogen Systems, 2017.

[33] J. Sun, G. Zheng, H.-W. Lee et al., "Formation of stable phosphorus-carbon bond for enhanced performance in black phosphorus nanoparticle-graphite composite battery anodes," Nano Letters, vol. 14, no. 8, pp. 4573-4580, 2014.

[34] J. Cabana, L. Monconduit, D. Larcher, and M. R. Palacín, "Beyond intercalation-based Li-ion batteries: the state of the art and challenges of electrode materials reacting through conversion reactions," Advanced Materials, vol. 22, no. 35, pp. E170-E192, 2010.

[35] V. Etacheri, R. Marom, R. Elazari, G. Salitra, and D. Aurbach, "Challenges in the development of advanced Li-ion batteries: a review," Energy \& Environmental Science, vol. 4, no. 9, pp. 32433262, 2011.

[36] H. Li, Z. Wang, L. Chen, and X. Huang, "Research on advanced materials for Li-ion batteries," Advanced Materials, vol. 21, no. 45, pp. 4593-4607, 2009.

[37] W. Li, C. Liang, W. Zhou et al., "Homogeneous and controllable Pt particles deposited on multi-wall carbon nanotubes as cathode catalyst for direct methanol fuel cells," Carbon, vol. 42, no. 2, pp. 436-439, 2004. 
[38] H. J. Kim, Y. S. Kim, M. H. Seo et al., "Highly improved oxygen reduction performance over $\mathrm{Pt} / \mathrm{C}$-dispersed nanowire network catalysts," Electrochemistry Communications, vol. 12, no. 1, pp. 32-35, 2010.

[39] A. Ziletti, A. Carvalho, D. K. Campbell, D. F. Coker, and A. H. Castro Neto, "Oxygen defects in phosphorene," Physical Review Letters, vol. 114, no. 4, Article ID 046801, 2015.

[40] Y. Cai, G. Zhang, and Y.-W. Zhang, "Layer-dependent band alignment and work function of few-layer phosphorene," Scientific Reports, vol. 4, article 6677, 2014.

[41] S. Chintalapati, L. Shen, Q. Xiong, and Y. P. Feng, "Magnetism in phosphorene: Interplay between vacancy and strain," Applied Physics Letters, vol. 107, no. 7, Article ID 072401, 2015.

[42] S. Zhang, J.-I. Hayashi, and C.-Z. Li, "Volatilisation and catalytic effects of alkali and alkaline earth metallic species during the pyrolysis and gasification of Victorian brown coal. Part IX. Effects of volatile-char interactions on char- $\mathrm{H} 2 \mathrm{O}$ and char-O2 reactivities," Fuel, vol. 90, no. 4, pp. 1655-1661, 2011.

[43] Y. Li, Z. Zhou, P. Shen, and Z. Chen, "Spin gapless semiconductor-metal-half-metal properties in nitrogendoped zigzag graphene nanoribbons," ACS Nano, vol. 3, no. 7, pp. 1952-1958, 2009.

[44] Y. Yagi, T. M. Briere, M. H. Sluiter, V. Kumar, A. A. Farajian, and Y. Kawazoe, "Stable geometries and magnetic properties of single-walled carbon nanotubes doped with $3 \mathrm{~d}$ transition metals: a first-principles study," Physical Review B, vol. 69, no. 7, pp. 428-433, 2015.

[45] K. F. Mak, C. Lee, J. Hone, J. Shan, and T. F. Heinz, "Atomically thin $\mathrm{MoS}_{2}$ : a new direct-gap semiconductor," Physical Review Letters, vol. 105, no. 13, Article ID 136805, 2010.

[46] J. He, K. Wu, R. Sa, Q. Li, and Y. Wei, "Magnetic properties of nonmetal atoms absorbed MoS2 monolayers," Applied Physics Letters, vol. 96, no. 8, Article ID 082504, 2010.

[47] C. Ataca and S. Ciraci, "Functionalization of single-layer MoS2 honeycomb structures," Journal of Physical Chemistry C, vol. 115, no. 27, pp. 13303-13311, 2011.

[48] H. Fang, S. Chuang, T. C. Chang, K. Takei, T. Takahashi, and A. Javey, "High-performance single layered WSe 2 p-FETs with chemically doped contacts," Nano Letters, vol. 12, no. 7, pp. 3788-3792, 2012.

[49] M. Thripuranthaka, C. S. Rout, and D. J. Late, "MoS2 nanoparticles and $\mathrm{h}-\mathrm{BN}$ nanosheets from direct exfoliation of bulk powder: one-step synthesis method," Materials Research Express, vol. 1, no. 3, Article ID 035038, 2014.

[50] C. Ataca and S. Ciraci, "Functionalization of BN honeycomb structure by adsorption and substitution of foreign atoms," Physical Review B - Condensed Matter and Materials Physics, vol. 82, no. 16, Article ID 165402, 2010.

[51] Y. Wang and Y. Ding, "First-principles study of the electronic and magnetic properties of 4-8 line-defect-embedded BN sheets decorated with transition metals," Annalen der Physik, vol. 526, no. 9-10, pp. 415-422, 2014

[52] L. Li, G. J. Ye, V. Tran et al., "Quantum oscillations in a twodimensional electron gas in black phosphorus thin films," Nature Nanotechnology, vol. 10, no. 7, pp. 608-613, 2015.

[53] Q. Wei and X. Peng, "Superior mechanical flexibility of phosphorene and few-layer black phosphorus," Applied Physics Letters, vol. 104, no. 25, Article ID 251915, 2014.

[54] L. Liang, J. Wang, W. Lin, B. G. Sumpter, V. Meunier, and M. Pan, "Electronic bandgap and edge reconstruction in phosphorene materials," Nano Letters, vol. 14, no. 11, pp. 6400-6406, 2014.
[55] J. Guan, Z. Zhu, and D. Tománek, "Phase coexistence and metalinsulator transition in few-layer phosphorene: a computational study," Physical Review Letters, vol. 113, no. 4, Article ID 046804, 2014.

[56] L. Kou, T. Frauenheim, and C. Chen, "Phosphorene as a superior gas sensor: selective adsorption and distinct $I-V$ response," Journal of Physical Chemistry Letters, vol. 5, no. 15, pp. 26752681, 2014.

[57] F. Xia, H. Wang, and Y. Jia, "Rediscovering black phosphorus as an anisotropic layered material for optoelectronics and electronics," Nature Communications, vol. 5, article 4458, 2014.

[58] M. Buscema, D. J. Groenendijk, G. A. Steele, H. S. J. Van Der Zant, and A. Castellanos-Gomez, "Photovoltaic effect in fewlayer black phosphorus PN junctions defined by local electrostatic gating," Nature Communications, vol. 5, article 4651, 2014.

[59] T. P. Kaloni, “Tuning the structural, electronic, and magnetic properties of germanene by the adsorption of $3 \mathrm{~d}$ transition metal atoms," Journal of Physical Chemistry C, vol. 118, no. 43, pp. 25200-25208, 2014.

[60] X. Peng, A. Copple, and Q. Wei, "Edge effects on the electronic properties of phosphorene nanoribbons," Journal of Applied Physics, vol. 116, no. 14, Article ID 144301, 2014.

[61] J. Son, A. Hashmi, and J. Hong, "Manipulation of $\mathrm{n}$ and $\mathrm{p}$ type dope black phosphorene layer: a first principles study," Current Applied Physics, vol. 16, no. 5, pp. 506-514, 2016.

[62] G. Wang, R. Pandey, and S. P. Karna, "Effects of extrinsic point defects in phosphorene: B, C, N, O, and F adatoms," Applied Physics Letters, vol. 106, no. 17, Article ID 173104, 2015.

[63] T. P. Kaloni, Y. C. Cheng, R. Faccio, and U. Schwingenschlögl, "Oxidation of monovacancies in graphene by oxygen molecules," Journal of Materials Chemistry, vol. 21, no. 45, pp. 18284-18288, 2011.

[64] Q. Feng, N. Tang, F. Liu et al., "Obtaining high localized spin magnetic moments by fluorination of reduced graphene oxide," ACS Nano, vol. 7, no. 8, pp. 6729-6734, 2013.

[65] T. P. Kaloni, M. Upadhyay Kahaly, R. Faccio, and U. Schwingenschlögl, "Modelling magnetism of $\mathrm{C}$ at $\mathrm{O}$ and $\mathrm{B}$ monovacancies in graphene," Carbon, vol. 64, no. 9, pp. 281-287, 2013.

[66] G. Wang, W. J. Slough, R. Pandey, and S. P. Karna, "Degradation of phosphorene in air: understanding at atomic level," $2 D$ Materials, vol. 3, no. 2, Article ID 025011, 2016.

[67] W. Sun, B. X. Ma, and J. F. Fan, "Physical properties of phosphorene surface adsorption with nonmetal atoms," Journal of Zhengzhou University (Natural Science Edition, vol. 4, pp. 47-51, 2015.

[68] R. Babar and M. Kabir, "Transition metal and vacancy defect complexes in phosphorene: a spintronic perspective," Journal of Physical Chemistry C, vol. 120, no. 27, pp. 14991-15000, 2016.

[69] C. X. Wang, C. Zhang, J. Jiang, and T. Rabczuk, "The effects of vacancy and oxidation on black phosphorus nanoresonators," Nanotechnology, vol. 28, no. 13, Article ID 135202, 2017. 

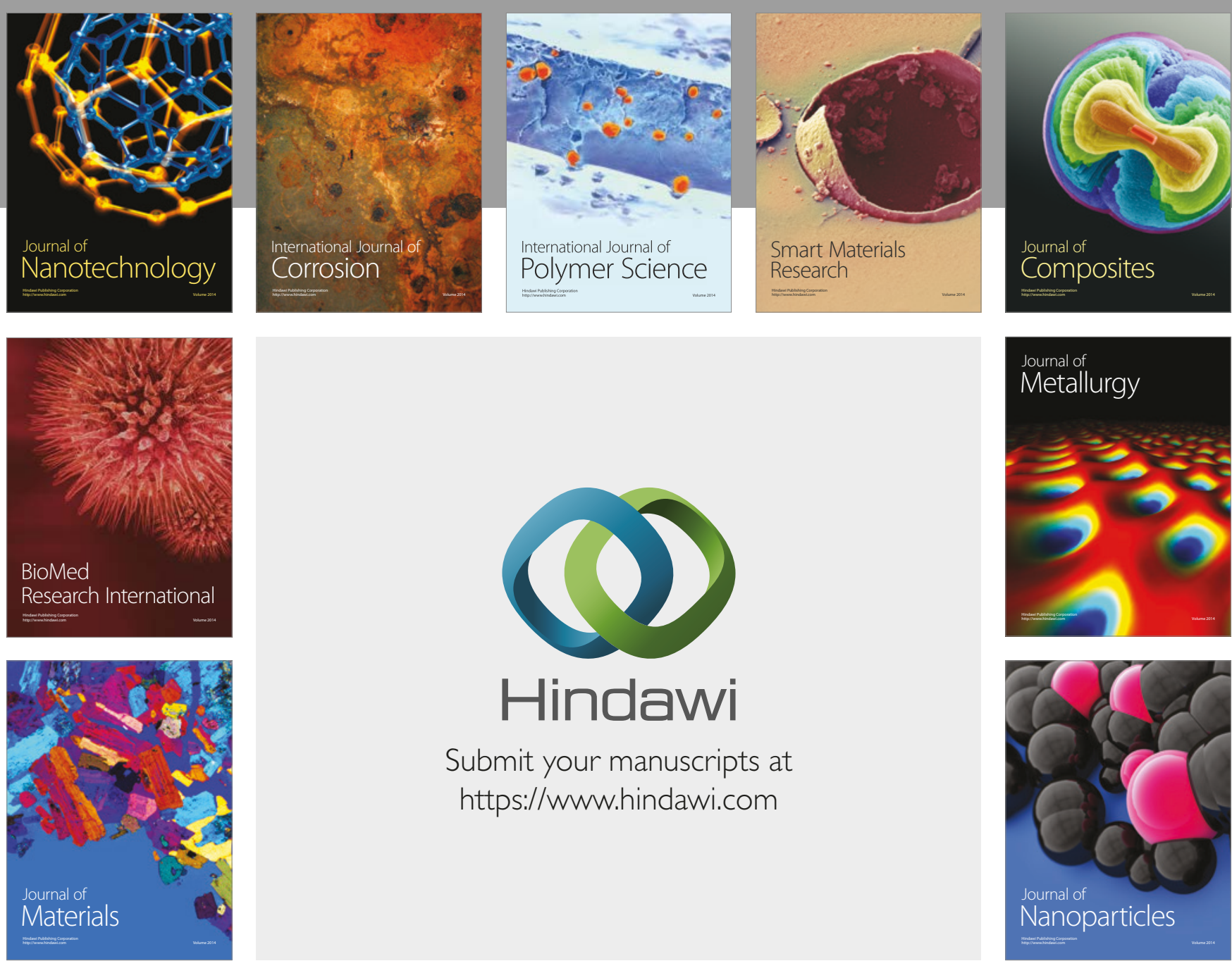

\section{Hindawi}

Submit your manuscripts at

https://www.hindawi.com
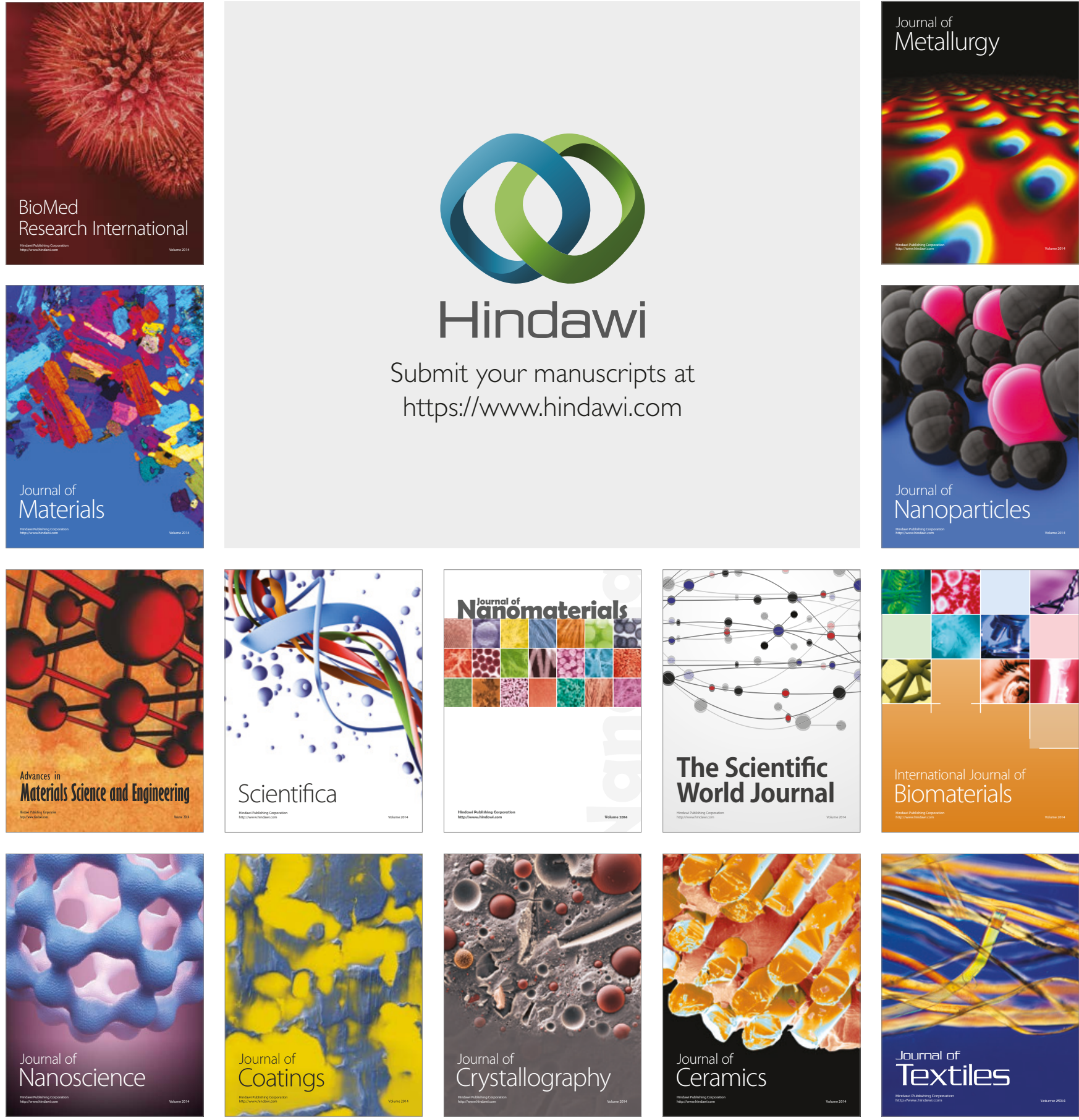

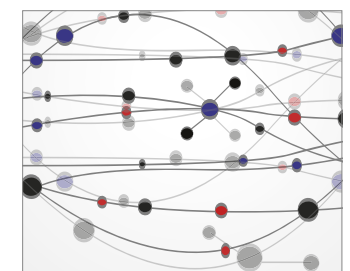

The Scientific World Journal
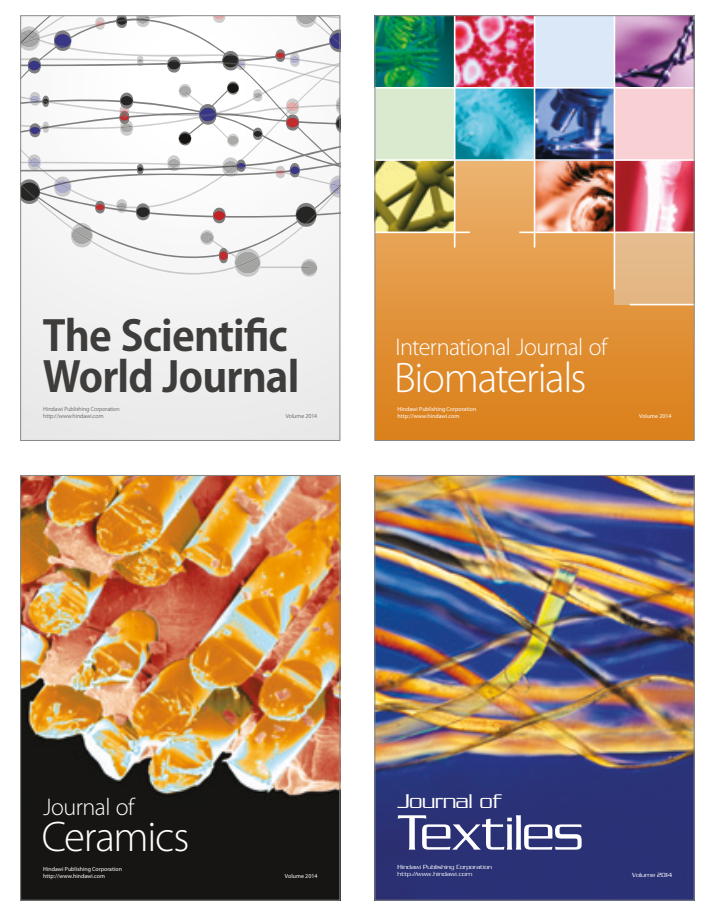